\title{
Standardization of concentrated antibodies for use in automated immunohistochemistry
}

\author{
Emily Ferreira Salles Pilar ${ }^{1 *}$ and Claudio Galleano Zettler ${ }^{2}$
}

*Correspondence: epilar@hcpa.edu.br

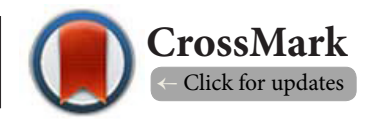

'Experimental Pathology Unit, Hospital de Clínicas de Porto Alegre (HCPA), Ramiro Barcelos, 2350 - Santa Cecilia, Porto Alegre - RS, 90035-007, Brazil.

${ }^{2}$ Department of Pathology, Santa Casa de Misericórdia de Porto Alegre, Universidade Federal de Ciências da Saúde de Porto Alegre (UFCSPA), Sarmento Leite, 245 - Centro Histórico, Porto Alegre - RS, 90050-170,Brazil.

\begin{abstract}
Background: Immunohistochemistry (IHC) is a method of identifying proteins in cells or tissues that is useful for diagnosis and research. In modern pathology, it has assumed an important supporting role in the molecular diagnosis of certain neoplasia, with an exponential contribution in personalized medicine. Automation in immunohistochemistry contributes to reduced test variability through standardization. The transition to automation is a process, and for a laboratory that already has a collection of stockconcentrated primary antibodies, ideally, these antibodies will be compatible with the chosen automated method, as antibodies are of high value to be discarded as a consequence of migration to automated immunohistochemistry.
\end{abstract}

Methods: 78 concentrated antibodies were tested for use in Ventana Medical Systems' Benchmark XT automation platform. Thirty-one human tissue samples containing the antigens of interest were used as positive control.

Results: All antibodies tested showed good performance, indicating the feasibility of using these antibodies concentrated in the automation platform in question. The protocol most frequently used was the one with antigenic retrieval with Cell Conditioning 1 for 60 minutes and incubation in the primary antibody for 32 minutes at $42^{\circ} \mathrm{C}$. The dilutions of the primary antibodies in automation ranged from 1:20 to 1:4000.

Conclusion: Under the aforementioned conditions, it was possible to take advantage of the portfolio of concentrated antibodies present in the laboratory at the time of transition from manual to automated immunohistochemistry.

Keywords: Automation, immunohistochemistry, standardization, concentrated antibodies

\section{Introduction}

Immunohistochemistry $(\mathrm{IHC})$ is a technique that is used to identify proteins in cells or tissue constituents through antigen-antibody interaction, and it represents an important tool in the identification and localization of a variety of antigens [1]. IHC stands at the interface between traditional pathology, pathology recognition based on microscopic analysis of morphology, and molecular diagnosis. In addition to the invaluable utility of IHC as a tool for classifying neoplasms in the era of personalized medicine, the method has become essential for the correct stratification of oncology patients for target-specific $[2,3]$.
In modern pathology, IHC has at least three main contributions: (1) diagnostic-mainly, the use of antibodies for the diagnosis of undifferentiated neoplasms, determination of primary site in metastatic diseases, and subtyping of neoplasias; (2) geneticsanalysis of gain or loss of protein expression due to changes in genes and the mutational state of certain biomarkers; and (3) therapeutic-through the analysis and quantification of tissue expression, IHC results can determine the best treatment option and predict response to a target-specific $[2,4]$.

Although IHC is a widely used method in diagnosis and research, there is a lack of standardization that may contribute to intra- and interlaboratory divergences $[4,5]$. Among the

(ㄷ) 2020 Pilar et al; licensee Herbert Publications Ltd. This is an Open Access article distributed under the terms of Creative Commons Attribution License (http://creativecommons.org/licenses/by/3.0). This permits unrestricted use, distribution, and reproduction in any medium, provided the original work is properly cited. 
challenges permeating $\mathrm{IHC}$ are pre-analytical factors such as fixation of the samples, analytical factors such as selection of the antibody (whether monoclonal or polyclonal), choice of detection system, use of controls, and endogenous protein and enzyme blockade [6-8].

It is not possible to standardize a single protocol in all laboratories, but it should be possible to standardize the protocols within a single laboratory, in order to ensure reproducibility. This requires strict adherence to the protocol during the performance of the technique. It is clear that performance consistency, just as in clinical analysis labs, can only be achieved by automation, given its inherent consistency and control $[6,9]$.

Among the main advantages of implementing IHC automation are the standardization of protocols and the possibility of increasing workload without compromising quality. Monitoring errors in processes with alarms for adverse situations, such as inadequate temperature and reagent volumes, expiration date control, and reagent inventory are also important $[10,11]$.

The kinetics during incubation in automation, heating, and evaporation control guarantee a uniform environment that leads to reproducible results, optimizing and accelerating the reactions [10]. Variations in reagent use flexibility in automation platforms gave rise to the terms "open" or "closed" system. Closed systems offer greater standardization but with less flexibility. Open-system platforms, in contrast, enable easy migration from a manual routine to an automated one through the flexibility of reagents, to include the use of manual routine antibodies and protocol customization; thus, open platforms are preferable in research $[10,11]$. Laboratories incorporating manual immunohistochemistry have concentrated antibodies in stock; however, in the transition to automation, these financially valuable reagents would be discarded. In this study, the laboratory already had a portfolio of antibodies and opted for a platform that accepts antibodies from other suppliers, prompting us to ask whether a good immunostaining with concentrated antibodies from other suppliers in the Benchmark automation platform XT-Ventana Medical Systems could be obtained. Thus, we will test the hypothesis that it will indeed be possible to use concentrated antibodies from other manufacturers, with satisfactory immunostaining, on Benchmark XT automation platform.

\section{Methods}

In this experimental study stock-concentrated antibodies from manufacturers ARP, Calbiochem, Dako, Diagnostic BioSystems (DBS) and Novocastra (Leica Biosystems) were tested on the Benchmark Ventana automation platform.

\section{Tissue samples}

The study was approved by the Ethics Committee in Research at the Universidade Federal de Ciências da Saúde de Porto Alegre (UFCSPA), opinion no. 332.213. Thirty one human samples (Table 1) fixed in 10\% neutral buffered formalin and embedded in paraffin were selected from the Pathology Department, Santa Casa de Misericórdia de Porto Alegre, based on the presence of the antigen of interest for antibody testing. All tissue samples used in this standardization were selected five years after the issuance of the report containing the anatomopathological diagnosis, without prejudice to the conclusion of the case. Samples were already part of a bank of positive tissue controls in the laboratory.

To test the protocols, the samples were cut to $2-\mu \mathrm{m}$ thickness, placed on StarFrost slides (Knittel Glass, Bielefeld, GER, ref. 9589), and individually labeled. The concentrated antibodies were stored, according to the manufacturer's instructions, at $2-8^{\circ} \mathrm{C}$ or in a freezer with automatic defrost from $-30^{\circ} \mathrm{C}$ to $-20^{\circ} \mathrm{C}$. The manufacturer's indication in the data sheet for antigen retrieval and dilution was verified, and an algorithm (Figure 1) was followed. Antigen retrieval was divided into three groups: (1) heat-induced epitope retrieval; (2) enzymatic antigen retrieval with Protease I $(0.38 \mathrm{mg} / \mathrm{mL}$ of alkaline protease in sodium azide of $0.01 \%$; Ventana Medical Systems, Oro Valley, AZ, EUA, ref. 760-2018); and (3) antigen retrieval would not be required.

\section{Manual technique}

The protocol that follows was used for all antibodies until the implementation of automation. Deparaffinization $\left(65^{\circ} \mathrm{C}\right)$ and antigen retrieval were performed using PT Link, Pre-Treatment Module for Tissue Specimens (Agilent), at a temperature of $97^{\circ} \mathrm{C}$ without using pressure, with appropriate Target Retrieval Solution for 30 minutes. Endogenous peroxidase blockade was done for all antibodies by Dual Endogenous Enzyme Block, contained in the Dako EnVision Dual Link SystemHRP (DAB) detection kit (K4065), for 10 minutes. Primary antibodies were diluted in Antibody Diluent, Background

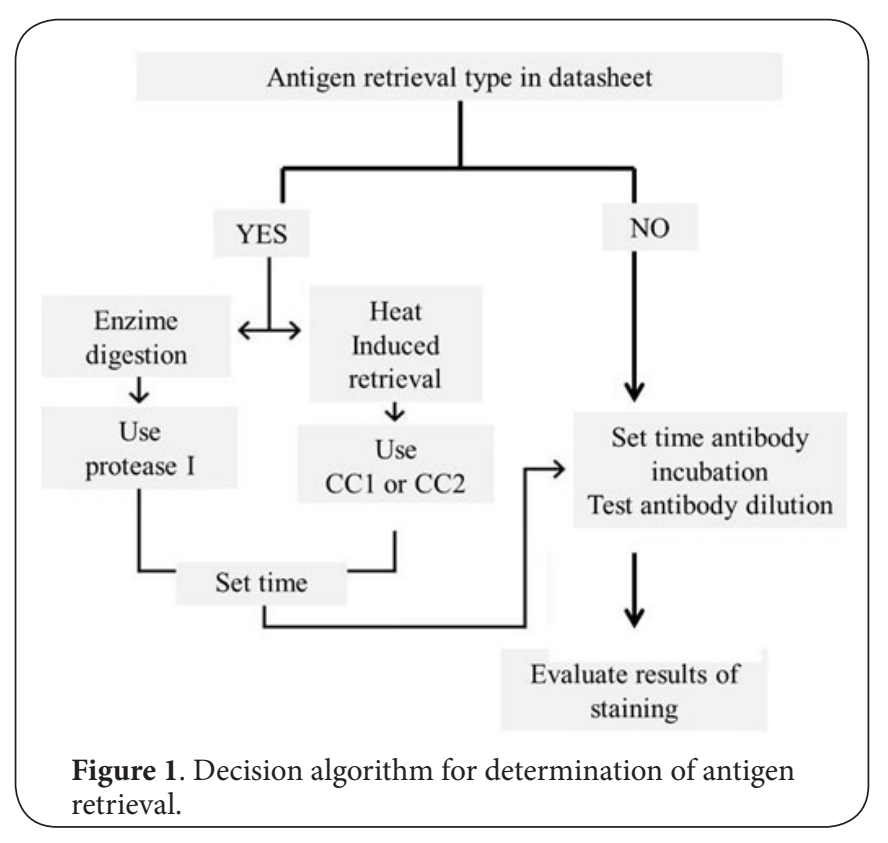


Pilar et al., Journal of Histology \& Histopathology 2020,

http://www.hoajonline.com/journals/pdf/2055-091X-7-4.pdf

Table 1. Samples used as a positive control.

\begin{tabular}{|c|c|c|}
\hline ID & Sample & Antibody \\
\hline 1 & Appendix & Desmin, Muscle actin, Smooth Muscle actin, Vimentin \\
\hline 2 & Breast & Ck7, Ck8/18, E-cadherin, Epithelial Membrane Antigen (EMA) \\
\hline 3 & Bone (Plasmocytoma) & Cd 38, Kappa Light Chains (KLC), Lambda Light chains (LLC) \\
\hline 4 & Breast carcinoma & Her2, Estrogen receptor, P53 \\
\hline 5 & Infected lung & Cytomegalovírus (CMV) \\
\hline 6 & Infected lymph node & Epstein Barr Vírus (EBV) \\
\hline 7 & Infected kidney & Polioma vírus \\
\hline 8 & Kidney & Collagen IV \\
\hline 9 & Large intestine & Cdx2, Carcinoembryonic Antigen (CEA), Ck20, Villin \\
\hline 10 & Liver & Alpha-1-fetoprotein, Hepatocyte specific antigen (HSA) \\
\hline 11 & Lung & Cd68 \\
\hline 12 & Lung adenocarcinoma & Napsin A \\
\hline 13 & Lymph node & $\begin{array}{l}\text { Blc2, Blc6, Cd7, Cd8, Cd10, Cd20, Cd21, Cd23, Granzime B, Leucocyte } \\
\text { common antigens (LCA) }\end{array}$ \\
\hline 14 & Lymph node (Anaplastic large cell lymphoma) & Alk 1 \\
\hline 15 & Lymph node (Lymphoblastic cell lymphoma) & Terminal Deoxynucleotidyl transferase (TDT) \\
\hline 16 & Lymph node (Hodgkin's lymphoma) & $\mathrm{Cd} 15, \mathrm{Cd} 30$ \\
\hline 17 & Medullary thyroid carcinoma & Calcitonin \\
\hline 18 & Neuroendocrine pancreatic carcinoma & Cd57, Chromogranin A, Neuron specific enolase (NSE), Synaptophysin \\
\hline 19 & Nerve (Peripheral nervous system) & Glial fibrillary acidic protein (GFAP), Neurofilament protein (NFP), S100 \\
\hline 20 & Ovarian serous papilliferous adenocarcinoma & Wilm's tumor 1 (WT1) \\
\hline 21 & Pancreas & Glucagon \\
\hline \multirow[t]{2}{*}{22} & Placenta & Annexin A1, Cd 31, Cd34, Chorionic Gonadotropin (HCG) \\
\hline & & Placental Alkaline Phosphatase (PLAP), Von Villenbrand Factor (VWF) \\
\hline 23 & Prostate & Alpha-methylacyl-CoA racemase (AMACR), Prostate Specific Antigen (PSA) \\
\hline 24 & Pituitary & $\begin{array}{l}\text { Adrenocorticotropin (ACTH), Follicle Stimulating Hormone (FSH), Prolactin } \\
(\mathrm{PRL})\end{array}$ \\
\hline 25 & Skeletal muscle & Myoglobin \\
\hline 26 & Skin & Ck 5/6, Ck Pan \\
\hline 27 & Skin (Melanoma) & Melan-A \\
\hline 28 & Small bowel (GIST) & Dog1 \\
\hline 29 & Testicle & Myeloperoxidase \\
\hline 30 & Thyroid & Thyroglobulin, Thyroid transcription Factor-1(TTF1) \\
\hline 31 & Tonsil & $\begin{array}{l}\text { B Cell Specific Octamer Binding Protein1 (BOB1), Immunoglobulin A(IgA), } \\
\text { Immunoglobulin D (IgD) Immunoglobulin G (IgG), Immunoglobulin M (IgM), } \\
\text { Ki67, Lysozyme, Multiple myeloma oncogene } 1 \text { (Mum1) }\end{array}$ \\
\hline
\end{tabular}

Reducing (ref. S3022), Dako Denmark A/S, and incubated overnight at $2-8^{\circ} \mathrm{C}$. Concentrations used in the manual routine are presented in Table 2, these data were used to compare concentrations of antibodies in the manual technique with those in the automated technique, and automation dilutions were also classified as lesser than, equal to, or greater than those used in the manual technique. The detection system used was Dako EnVision + Dual Link System-HRP (K4065) with Dab Chromogen, following manufacturer's instructions; the counterstaining step was performed with Harris' hematoxylin, followed by washing in $1 \%$ ammonium hydroxide. Washing buffer was phosphate-buffered saline, pH 7.0.

\section{Automated IHC staining and evaluation}

Seventy-eight concentrate antibodies were tested (Table 2), immunohistochemistry reactions were performed on VENTANA BenchMark XT (Ventana Medical Systems, Oro Valley, AZ, EUA) equipment, and the detection system used was UltraView Universal DAB Detection Kit (Ventana Medical Systems, Oro Valley, AZ, EUA, ref. 760-500). Endogenous peroxidase blockade was done for all antibodies in automation it is performed by the ultraView Universal DAB reagent Inhibitor contains $3 \%$ hydrogen peroxide solution, time is not provided by the manufacturer. The counterstain reagent was Hematoxylin II (Ventana Medical Systems, Oro Valley, AZ, EUA, ref. 790-2208), 
Pilar et al., Journal of Histology \& Histopathology 2020,

http://www.hoajonline.com/journals/pdf/2055-091X-7-4.pdf

Table 2. Primary antibodies tested in automation.

\begin{tabular}{|c|c|c|c|c|c|}
\hline Antibody & Clonality & Cellular localization & Lot & Supplier & Manual dilution \\
\hline ACTH & $02 \mathrm{~A} 3$ & Cytoplasmic & 10044413 & DAKO & $1: 1500$ \\
\hline ALK Protein & ALK1 & Cytoplasmic/nucleus & 00056980 & DAKO & $1: 300$ \\
\hline Alpha-1- Fetoprotein & Polyclonal & Cytoplasmic & 00058169 & DAKO & $1: 500$ \\
\hline AMACR & EPMU1 & Cytoplasmic & 6005488 & NOVOCASTRA & $1: 500$ \\
\hline $\mathrm{Bcl} 2$ & 124 & Cytoplasmic & 00056477 & DAKO & $1: 300$ \\
\hline Bcl 6 & PG-B6p & Nucleus & 00053818 & DAKO & $1: 200$ \\
\hline BOB 1 & TG14 & Cytoplasmic/nucleus & 6003030 & NOVOCASTRA & $1: 200$ \\
\hline Calcitonin & CL1948 & Cytoplasmic & 6003520 & NOVOCASTRA & $1: 2000$ \\
\hline CEA & Polyclonal & Cytoplasmic & 00034436 & DAKO & $1: 500$ \\
\hline $\mathrm{CD} \times 2$ & AMT 28 & Nucleus & 6000213 & NOVOCASTRA & $1: 700$ \\
\hline $\mathrm{CD} 7$ & СBC. 37 & Membrane & 00053089 & DAKO & $1: 50$ \\
\hline CD 8 & $\mathrm{C} 8 / 144 \mathrm{~B}$ & Membrane & 00056474 & DAKO & $1: 300$ \\
\hline CD10 & NCL-L-CD10-270 & Membrane & 6019294 & NOVOCASTRA & $1: 25$ \\
\hline CD 15 & BY87 & Membrane & 6015073 & NOVOCASTRA & $1: 200$ \\
\hline $\mathrm{CD} 20$ & L 26 & Membrane & 00050873 & DAKO & $1: 600$ \\
\hline $\mathrm{CD} 21$ & $1 \mathrm{~F} 8$ & Membrane & 00080760 & DAKO & $1: 200$ \\
\hline $\mathrm{CD} 23$ & MHM6 & Membrane & 00049836 & DAKO & $1: 200$ \\
\hline $\mathrm{CD} 30$ & Ber - H2 & Membrane & 00057005 & DAKO & $1: 700$ \\
\hline CD 31 & JC70A & Cytoplasmic/membrane & 00051102 & DAKO & $1: 100$ \\
\hline CD 34 & QBEnd- 10 & Membrane & 00053768 & DAKO & $1: 100$ \\
\hline $\mathrm{CD} 38$ & SPC32 & Membrane & 6006807 & NOVOCASTRA & $1: 100$ \\
\hline CD 57 & TB 01 & Membrane & 00061124 & DAKO & $1: 200$ \\
\hline CD 68 & PGM1 & Cytoplasmic & 00058931 & DAKO & $1: 500$ \\
\hline HCG & Polyclonal & Cytoplasmic & 00053071 & DAKO & $1: 1500$ \\
\hline Chromogranin A & DAK - A3 & Cytoplasmic & 00052696 & DAKO & $1: 1000$ \\
\hline Cytokeratin 5/6 & $\mathrm{D} 5 / 16 \mathrm{~B} 4$ & Cytoplasmic & 00060846 & DAKO & $1: 400$ \\
\hline Cytokeratin 7 & OV-TL 12/30 & Cytoplasmic & 00050559 & DAKO & $1: 800$ \\
\hline Cytokeratin 8/18 & $5 \mathrm{D} 3$ & Cytoplasmic & L106751 & NOVOCASTRA & $1: 700$ \\
\hline Cytokeratin 20 & Ks 20.8 & Cytoplasmic & 00057249 & DAKO & $1: 700$ \\
\hline CKPAN & MNF 116 & Cytoplasmic & 00048696 & DAKO & $1: 200$ \\
\hline CMV & $\mathrm{CCH} 2+\mathrm{DDG} 9$ & Nucleus & 00057285 & DAKO & $1: 1000$ \\
\hline Collagen IV & CIV & Membrane & 00050818 & DAKO & $1: 50$ \\
\hline Desmin & D 33 & Cytoplasmic & 00052230 & DAKO & $1: 300$ \\
\hline DOG-1 & K9 & Cytoplasmic & 6007637 & NOVOCASTRA & $1: 100$ \\
\hline EBV & CS.1-4 & Cytoplasmic & 00055677 & DAKO & $1: 700$ \\
\hline E-cadherin & $\mathrm{NCH}-38$ & Membrane & 10042951 & DAKO & $1: 400$ \\
\hline EMA & E29 & Cytoplasmic/membrane & 00051982 & DAKO & $1: 300$ \\
\hline Estrogen Receptor & SP1 & Nucleus & 00050839 & DAKO & $1: 400$ \\
\hline FSH & $\mathrm{C} 10$ & Cytoplasmic & 10039598 & DAKO & $1: 800$ \\
\hline Granzime B & GrB-7 & Cytoplasmic & 00055619 & DAKO & $1: 300$ \\
\hline GFAP & $6 \mathrm{~F} 2$ & Cytoplasmic & 00062411 & DAKO & $1: 400$ \\
\hline Glucagon & Polyclonal & Cytoplasmic & 10040085 & DAKO & $1: 500$ \\
\hline Her2 & SP3 & Membrane & $\mathrm{R} 026$ & DBS & $1: 1500$ \\
\hline
\end{tabular}


Continuation of Table 2.

\begin{tabular}{|c|c|c|c|c|c|}
\hline Antibody & Clonality & Cellular localization & Lot & Supplier & Manual dilution \\
\hline Hepatocyte & OCH1E5 & Cytoplasmic & 00061010 & DAKO & $1: 400$ \\
\hline $\operatorname{IgA}$ & N1CLA. & Cytoplasmic & 6008931 & NOVOCASTRA & $1: 10000$ \\
\hline $\operatorname{IgD}$ & DRN1C & Cytoplasmic & 6009435 & NOVOCASTRA & $1: 10000$ \\
\hline $\operatorname{IgG}$ & RWP49 & Cytoplasmic & 6009436 & NOVOCASTRA & $1: 10000$ \\
\hline $\operatorname{IgM}$ & $8 \mathrm{H} 6$ & Cytoplasmic & 6007528 & NOVOCASTRA & $1: 10000$ \\
\hline KLC & $\mathrm{CH} 15$ & Cytoplasmic & 6006134 & NOVOCASTRA & $1: 3000$ \\
\hline Ki-67 & MIB-1 & Nucleus & 00079939 & DAKO & $1: 400$ \\
\hline LLC & SHL53 & Cytoplasmic & 6005595 & NOVOCASTRA & $1: 10000$ \\
\hline LCA & $2 \mathrm{~B} 11+\mathrm{PD} 7 / 26$ & Membrane & 00055808 & DAKO & $1: 600$ \\
\hline Lysozyme & Polyclonal & Cytoplasmic & 00051100 & DAKO & $1: 1500$ \\
\hline Melan-A & A 103 & Cytoplasmic & 00051087 & DAKO & $1: 500$ \\
\hline Muscle Actin & HHF35 & Cytoplasmic & 10042823 & DAKO & $1: 500$ \\
\hline Myeloperoxidase & 59A5 & Cytoplasmic & 6000030 & NOVOCASTRA & $1: 300$ \\
\hline MUM 1 & muml p & Nucleus/cytoplasmic & 00060192 & DAKO & $1: 200$ \\
\hline Napsin A & $01-0221$ & Cytoplasmic & 60828 & ARP & $1: 500$ \\
\hline Myoglobin & MYO18 & Cytoplasmic & 6008887 & NOVOCASTRA & $1: 500$ \\
\hline NFP & $2 \mathrm{~F} 11$ & Cytoplasmic & 00085150 & DAKO & $1: 200$ \\
\hline NSE & $\begin{array}{l}\mathrm{BBS} / \mathrm{NC} / \mathrm{V} \\
\mathrm{I}-\mathrm{H} 14\end{array}$ & Cytoplasmic & 00051206 & DAKO & $1: 600$ \\
\hline P53 & DO-7 & Nucleus & 00050255 & DAKO & $1: 800$ \\
\hline P63 & 7JUL & Nucleus & 6010555 & NOVOCASTRA & $1: 200$ \\
\hline PLAP & $8 \mathrm{~A} 9$ & Cytoplasmic & 00051152 & DAKO & $1: 200$ \\
\hline Polioma Vírus & PAb416 & Nucleus & D00073537 & CALBIOCHEM & $1: 200$ \\
\hline Prolactin & Polyclonal & Cytoplasmic & 10040256 & DAKO & $1: 1500$ \\
\hline PSA & ER-PR8 & Cytoplasmic & 00051198 & DAKO & $1: 900$ \\
\hline PSMA & $3 \mathrm{E} 6$ & Cytoplasmic & 10042956 & DAKO & $1: 900$ \\
\hline Synaptophysin & sy-38 & Cytoplasmic & 00064754 & DAKO & $1: 300$ \\
\hline S100 & Polyclonal & Nucleus/cytoplasmic & 00048024 & DAKO & $1: 3500$ \\
\hline Smooth Muscle Actin & $1 \mathrm{~A} 4$ & Cytoplasmic & 00059322 & DAKO & $1: 500$ \\
\hline TDT & SEN28 & Nucleus & 6004211 & NOVOCASTRA & $1: 100$ \\
\hline Thyroglobulin & DAK-Tg6 & Cytoplasmic & 00051933 & DAKO & $1: 1000$ \\
\hline TTF1 & SPT24 & Nucleus & 6011867 & NOVOCASTRA & $1: 1100$ \\
\hline VWF & F8-86 & Cytoplasmic & 00052965 & DAKO & $1: 100$ \\
\hline Villin & CWWB1 & Cytoplasmic/membrane & 6003038 & NOVOCASTRA & $1: 2500$ \\
\hline Vimentin & V9 & Cytoplasmic & 00051105 & DAKO & $1: 3000$ \\
\hline Wilms' Tumor 1 & $6 \mathrm{~F}-\mathrm{H} 2$ & Nucleus & 10042947 & DAKO & $1: 400$ \\
\hline
\end{tabular}


Pilar et al., Journal of Histology \& Histopathology 2020,

a modified Mayer's hematoxylin, time of counterstaining in hematoxylin II was defined according to the location of the antigen, with the objective of not masking nuclear antigens with Dab chromogen in the nucleus of the cells; for nuclear antigens, staining was done for 8 minutes, and for membrane antigens and cytoplasm, 12 minutes. All antibodies used were valid during the antibody testing period from January to December 2012.

Pathologist analysis used the following criteria: (a) specific reaction with the antigen, classified as 0 (absent), 1 (weak), 2 (satisfactory), or 3 (strong); (b) technical quality, classified as 0 (unsatisfactory), 1 (regular), or 2 (satisfactory); and (c) background color, classified as 0 (absent), 1 (low), or 2 (present). During microscopic evaluation by the pathologist when the staining was weak, even after an increase in the antigenic recovery time and the concentration of the primary antibody, the Amplification Kit (Ventana Medical Systems, Oro Valley, AZ, EUA, ref. 760-080) was added to the protocol. The Amplification Kit (Table 3) may be used in conjunction with
VENTANA detection kits to increase the signal intensity of weak-staining primary antibodies. After each run in the equipment the quality of the immunostaining was evaluated by the pathologist and this algorithm was followed for the decision making regarding changes in the protocols. When the result was considered unsatisfactory for any of the above reasons, the algorithm (Figure 2) for protocol change was followed. After defining the best protocol, each slide was digitalized using an Olympus BX51 microscope (Olympus Optical Co., Tokyo, Japan) connected to a digital color camera (Q-Color 5, Olympus); QCapture software was used for image capture.

\section{Results}

Protocols of 78 primary concentrated antibodies for automated use were standardized and are presented in Table 3. Immunostaining in automation photomicrographs are presented in Figures 3-6.

Regarding the types of antigenic retrieval, most of the antibodies benefited from an antigen retrieval in the Cell

Table 3. Protocols of concentrate antibodies for Ventana Benchmark XT- Automation.

\begin{tabular}{|c|c|c|c|c|c|c|c|}
\hline Antibody & $\begin{array}{l}\text { Antigen } \\
\text { retrieval } \\
\text { (min) }\end{array}$ & Incubation & Dilution & Antibody & $\begin{array}{l}\text { Antigen } \\
\text { retrieval } \\
(\mathrm{min})\end{array}$ & Incubation & Dilution \\
\hline ACTH & Not & $32 \mathrm{~min}$ & $1: 1000$ & Granzime B & $90 \mathrm{CC} 1$ & $90 \mathrm{~min}$ & $1: 50$ \\
\hline ALK Protein & $60 \mathrm{CCl}$ & $32 \mathrm{~min}$ & $1: 250$ & GFAP & $60 \mathrm{CC} 1$ & $32 \mathrm{~min}$ & $1: 500$ \\
\hline$\alpha-1-$ Fetoprotein & Not & $32 \min$ & $1: 50$ & Glucagon & Not & $32 \min$ & $1: 200$ \\
\hline AMACR & $60 \mathrm{CC} 1$ & $32 \mathrm{~min}$ & $1: 100$ & Her2 & $60 \mathrm{CC} 1$ & $32 \mathrm{~min}$ & $1: 500$ \\
\hline $\mathrm{Bcl} 2$ & $90 \mathrm{CC} 1$ & $90 \mathrm{~min}$ & $1: 25^{\star}$ & Hepatocyte & $60 \mathrm{CC} 1$ & $32 \mathrm{~min}$ & $1: 300$ \\
\hline $\mathrm{Bcl} 6$ & $90 \mathrm{CC} 1$ & $90 \mathrm{~min}$ & $1: 50^{*}$ & $\operatorname{IgA}$ & $60 \mathrm{CC} 1$ & $32 \mathrm{~min}$ & $1: 200$ \\
\hline BOB 1 & $90 \mathrm{CC} 1$ & $90 \mathrm{~min}$ & $1: 25^{\star}$ & $\operatorname{IgD}$ & $60 \mathrm{CC} 1$ & $32 \min$ & $1: 50$ \\
\hline Calcitonin & $4 \mathrm{P} 1$ & $32 \mathrm{~min}$ & $1: 300$ & $\operatorname{IgG}$ & $60 \mathrm{CC} 1$ & $32 \mathrm{~min}$ & $1: 1000$ \\
\hline CEA & $60 \mathrm{CC} 1$ & $32 \mathrm{~min}$ & $1: 1000$ & $\operatorname{IgM}$ & $60 \mathrm{CC} 1$ & $32 \mathrm{~min}$ & $1: 400$ \\
\hline $\mathrm{CDX} 2$ & $90 \mathrm{CC} 1$ & $90 \mathrm{~min}$ & $1: 25^{\star}$ & KLC & $60 \mathrm{CC} 1$ & $32 \mathrm{~min}$ & $1: 2000$ \\
\hline $\mathrm{CD} 7$ & $60 \mathrm{CC} 1$ & $32 \mathrm{~min}$ & $1: 50$ & $\mathrm{Ki}-67$ & $60 \mathrm{CC} 1$ & $32 \mathrm{~min}$ & $1: 50$ \\
\hline CD 8 & $60 \mathrm{CC} 1$ & $32 \mathrm{~min}$ & $1: 1000$ & LLC & $60 \mathrm{CC} 1$ & $32 \mathrm{~min}$ & $1: 2000$ \\
\hline CD10 & $60 \mathrm{CC} 1$ & $32 \mathrm{~min}$ & $1: 20$ & LCA & $60 \mathrm{CC} 1$ & $32 \mathrm{~min}$ & $1: 500$ \\
\hline CD 15 & $84 \mathrm{CC} 2$ & $60 \mathrm{~min}$ & $1: 25$ & Lysozyme & Not & $60 \mathrm{~min}$ & $1: 500$ \\
\hline CD 20 & $60 \mathrm{CC} 1$ & $32 \mathrm{~min}$ & $1: 500$ & Melan-A & $60 \mathrm{CC} 1$ & $32 \mathrm{~min}$ & $1: 100$ \\
\hline CD 21 & $4 \mathrm{P} 1$ & $60 \mathrm{~min}$ & $1: 25^{\star}$ & Muscle Actin & $60 \mathrm{CC} 1$ & $32 \min$ & $1: 300$ \\
\hline CD 23 & $60 \mathrm{CC} 1$ & $32 \mathrm{~min}$ & $1: 150$ & Myeloperoxidase & $60 \mathrm{CC} 1$ & $32 \mathrm{~min}$ & $1: 300$ \\
\hline CD 30 & $90 \mathrm{CC} 1$ & $60 \mathrm{~min}$ & $1: 30$ & MUM 1 & $60 \mathrm{CC} 1$ & $32 \mathrm{~min}$ & $1: 100$ \\
\hline CD 31 & $60 \mathrm{CC} 1$ & $32 \mathrm{~min}$ & $1: 100$ & Napsin A & $60 \mathrm{CC} 1$ & $32 \mathrm{~min}$ & $1: 500$ \\
\hline CD 34 & $90 \mathrm{CC} 1$ & $32 \mathrm{~min}$ & $1: 100$ & Myoglobin & $60 \mathrm{CC} 1$ & $60 \mathrm{~min}$ & $1: 400$ \\
\hline CD 38 & $60 \mathrm{CC} 1$ & $32 \mathrm{~min}$ & $1: 400$ & NFP & $60 \mathrm{CC} 1$ & $32 \mathrm{~min}$ & $1: 50$ \\
\hline CD 57 & $60 \mathrm{CC} 1$ & $32 \min$ & $1: 100$ & NSE & $60 \mathrm{CC} 1$ & $32 \min$ & $1: 500$ \\
\hline CD 68 & $60 \mathrm{CC} 1$ & $32 \mathrm{~min}$ & $1: 300$ & P53 & $60 \mathrm{CC} 1$ & $32 \mathrm{~min}$ & $1: 200$ \\
\hline HCG & $60 \mathrm{CC} 1$ & $32 \mathrm{~min}$ & $1: 4000$ & P63 & $60 \mathrm{CC} 1$ & $32 \mathrm{~min}$ & $1: 50$ \\
\hline Chromogranin A & $60 \mathrm{CC} 1$ & $32 \mathrm{~min}$ & $1: 250$ & PLAP & $60 \mathrm{CC} 1$ & $32 \mathrm{~min}$ & $1: 100$ \\
\hline
\end{tabular}


Pilar et al., Journal of Histology \& Histopathology 2020,

http://www.hoajonline.com/journals/pdf/2055-091X-7-4.pdf

Continuation of Table 3.

\begin{tabular}{|c|c|c|c|c|c|c|c|}
\hline Antibody & $\begin{array}{l}\text { Antigen } \\
\text { retrieval } \\
(\mathrm{min})\end{array}$ & Incubation & Dilution & Antibody & $\begin{array}{l}\text { Antigen } \\
\text { retrieval } \\
(\mathrm{min})\end{array}$ & Incubation & Dilution \\
\hline Cytokeratin 5/6 & $60 \mathrm{CC} 1$ & $32 \mathrm{~min}$ & $1: 200$ & Polioma Vírus & $60 \mathrm{CC} 1$ & $32 \mathrm{~min}$ & $1: 500$ \\
\hline Cytokeratin 7 & $60 \mathrm{CC} 1$ & $32 \mathrm{~min}$ & $1: 400$ & Prolactin & Not & $32 \mathrm{~min}$ & $1: 1250$ \\
\hline Cytokeratin 8/18 & $60 \mathrm{CC} 1$ & $32 \mathrm{~min}$ & $1: 350$ & PSA & Not & $32 \mathrm{~min}$ & $1: 100$ \\
\hline Cytokeratin 20 & $60 \mathrm{CC} 1$ & $32 \mathrm{~min}$ & $1: 350$ & PSMA & $90 \mathrm{CC} 1$ & $90 \mathrm{~min}$ & $1: 200$ \\
\hline CKPAN & $60 \mathrm{CC} 1$ & $32 \mathrm{~min}$ & $1: 100$ & Synaptophysin & $90 \mathrm{CC} 1$ & $90 \mathrm{~min}$ & $1: 50^{*}$ \\
\hline CMV & $4 \mathrm{P} 1$ & $32 \mathrm{~min}$ & $1: 800$ & S100 & $60 \mathrm{CC} 1$ & $32 \mathrm{~min}$ & $1: 2000$ \\
\hline Collagen IV & $90 \mathrm{CC} 1$ & $32 \mathrm{~min}$ & $1: 100$ & Smooth Muscle Actin & $60 \mathrm{CC} 1$ & $32 \mathrm{~min}$ & $1: 500$ \\
\hline Desmin & $60 \mathrm{CC} 1$ & $32 \mathrm{~min}$ & $1: 150$ & TDT & $60 \mathrm{CC} 1$ & $32 \mathrm{~min}$ & $1: 200$ \\
\hline DOG-1 & $90 \mathrm{CC} 1$ & $32 \mathrm{~min}$ & $1: 100$ & Thyroglobulin & Not & $32 \mathrm{~min}$ & $1: 500$ \\
\hline EBV & $60 \mathrm{CC} 1$ & $32 \mathrm{~min}$ & $1: 600$ & TTF-1 & $60 \mathrm{CC} 1$ & $32 \mathrm{~min}$ & $1: 600$ \\
\hline E-cadherin & $60 \mathrm{CC} 1$ & $32 \mathrm{~min}$ & $1: 150$ & VWF & $4 \mathrm{P} 1$ & $32 \mathrm{~min}$ & $1: 50$ \\
\hline EMA & $60 \mathrm{CC} 1$ & $32 \mathrm{~min}$ & $1: 100$ & Villin & $60 \mathrm{CC} 1$ & $32 \mathrm{~min}$ & $1: 300$ \\
\hline Estrogen Receptor & $90 \mathrm{CC} 1$ & $32 \mathrm{~min}$ & $1: 50$ & Vimentin & $60 \mathrm{CC} 1$ & $32 \mathrm{~min}$ & $1: 2000$ \\
\hline FSH & $4 \mathrm{P} 1$ & $32 \mathrm{~min}$ & $1: 100$ & Wilms' Tumor 1 & $8 \mathrm{P} 1$ & $90 \mathrm{~min}$ & $1: 50$ \\
\hline
\end{tabular}

Abbreviations: Ck: Cytokeratin; HMV: High Molecular Weight; AR: Antigen retrieval; CC1: Cell Conditioning 1; P1: Protease I; CC2: Cell Conditioning 2 * Use amplification kit.

Conditioning 1 buffer (Table 4). The most commonly used protocol was with antigen retrieval for 60 minutes and incubation time in the primary antibody for 32 minutes at $42^{\circ} \mathrm{C}$. All the algorithms and figures presented here were developed by the authors.

Incubation times with the primary antibody were $32 \mathrm{~min}$ utes $(83 \%), 60$ minutes $(6.4 \%)$, and 90 minutes $(10.2 \%)$. The dilutions of the primary antibodies ranged from 1:20 to 1:4000, while in the manual IHC method, dilutions ranged from 1:25 to $1: 10.000$. We observed that most antibodies (83\%) were used at lower (more concentrated) dilutions, while only $16 \%$ of antibodies could be used at the same or larger (less concentrated) dilutions than in the manual technique (Figure 7).

\section{Discussion}

All antibodies showed positive immunostaining, which indicates the feasibility of using the laboratory's in-stock concentrates when automation is implemented. The use of these reagents (concentrated antibodies) acquired and in stock represents savings for the laboratory, since their disposal, due to the mandatory purchase of antibodies ready for use from the Ventana, would represent an even greater expense as a result of the introduction of automation. In general, the antibodies in the automation platform needed to be more concentrated relative to the dilutions that were used in the manual routine. This may be due to the incubation time of the antibodies, which in the manual technique is at least 14 hours $\left(2-8^{\circ} \mathrm{C}\right)$ and in the automated, at most 90 minutes. In the study by Gedda et al. (2010), using real-time immunohistochemistry, the authors propose a direct and quantitative antigen-antibody interaction analysis, indicating that when
Table 4. Antigen Retrieval type.

\begin{tabular}{lll}
\hline Antigen retrieval & Antibodies & \% \\
\hline HIER $^{*}$ - Cell Conditioning 1 & 64 & 82.1 \\
HIER $^{*}$ Cell Conditioning 2 & 1 & 1.2 \\
Enzymatic epitope retrieval & 6 & 7.7 \\
Not required & 7 & 9 \\
\hline
\end{tabular}

${ }^{\star}$ HIER- Heat induced epitope retrieval

using incubations as recommended by many manufacturers for 30 minutes, the primary antibody had been linked to less than $10 \%$ of available sites. In addition, the authors point out that slight variations in incubation time and antibody concentration strongly affect the amount of bound antibody [12]. In our results, we observed that for more than $90 \%$ of the antibodies it was necessary to use some method of antigenic recovery, which is expected, since formalin (fixator commonly used in pathological anatomy) is a cross-link fixative, which can lead to loss of immunoreactivity, which can be restored by using the antigenic recovery technique [13]. Formalin's ability to cross-link is potentially deleterious to antigenic strutucture, however there are some epitopes that are not significantly affected. Despite the fixation of all samples used as positive controls in formalin, for 7 antibodies, it was not necessary to use the antigen retrieval. Perhaps this preservation of reactivity may be related to the fact that some epitopes are irreversibly modified by formalin while others are not [14]. Regarding the sensitivity of epitopes to formalin they can be classified into three main groups: formalin-resistant epitopes, highly formalin-sensitive epitopes and epitopes with a timedependent sensitivity to formalin fixation [15].

Some issues became evident during the process of transi- 


\section{Evaluate results of staining}

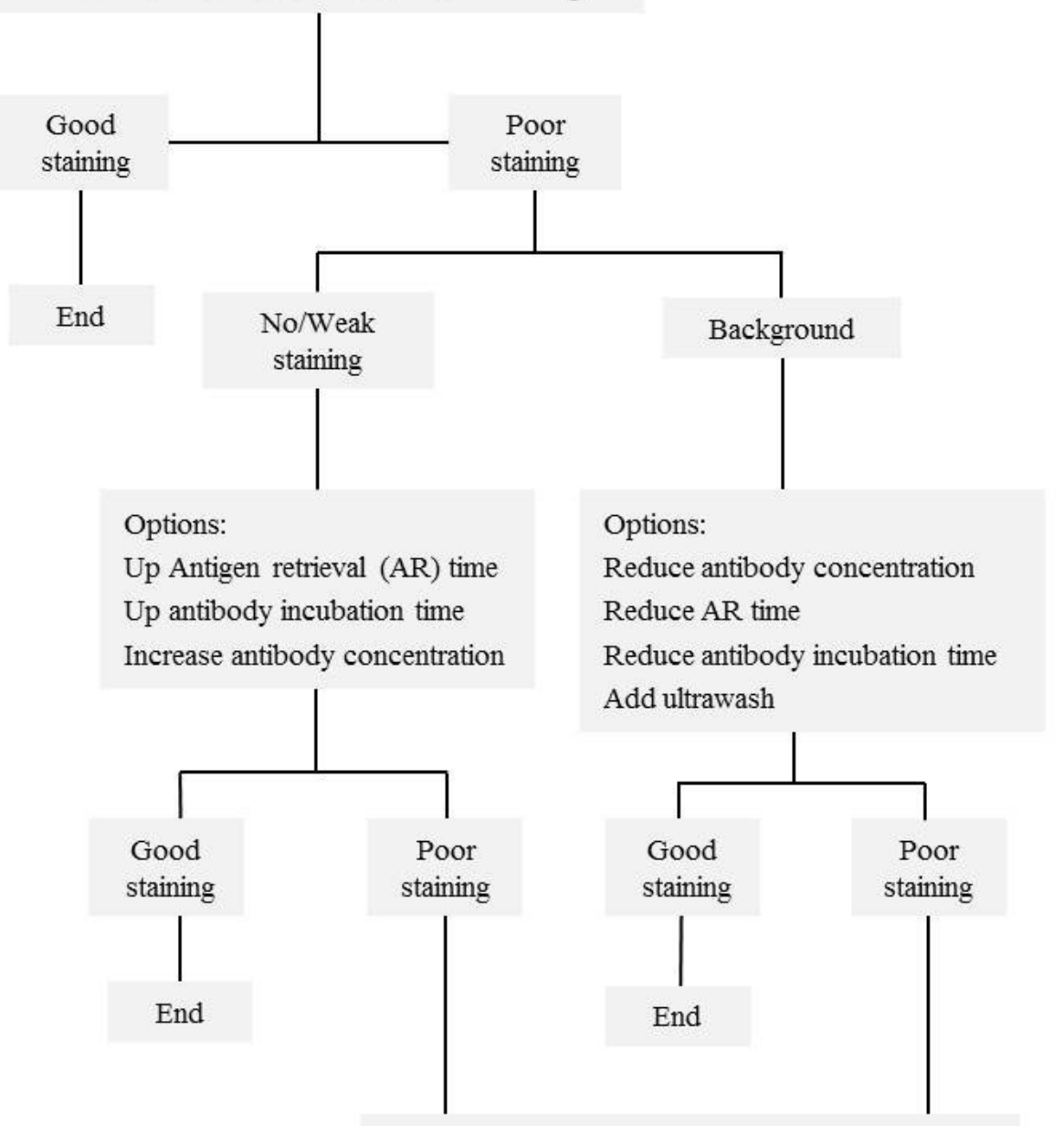

Options for change antigen retrieval type
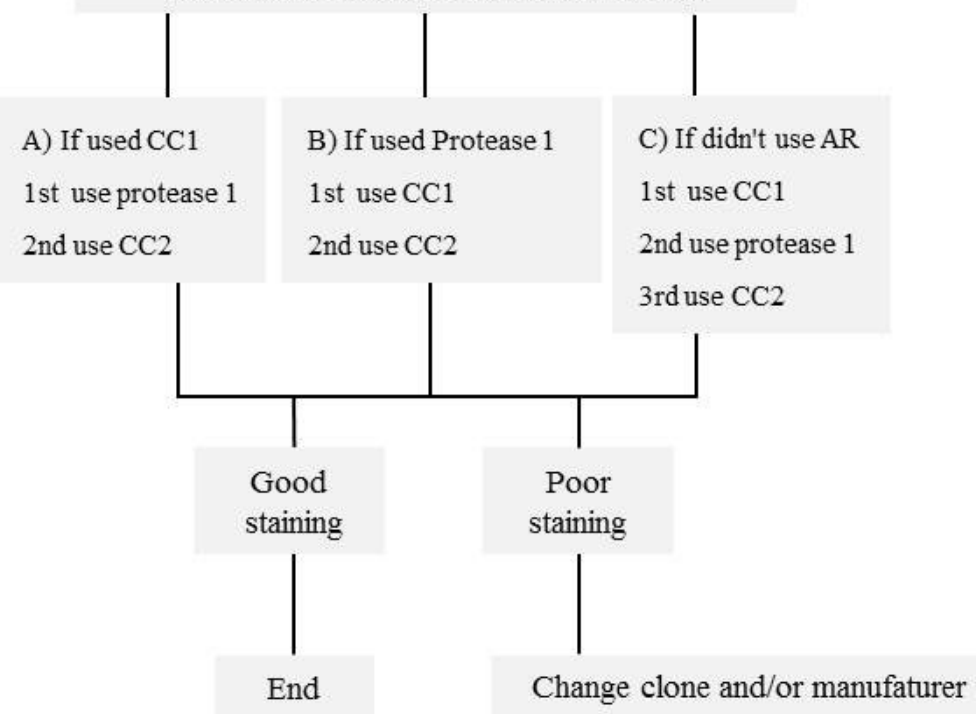

Figure 2. Algorithm for evaluate results. 


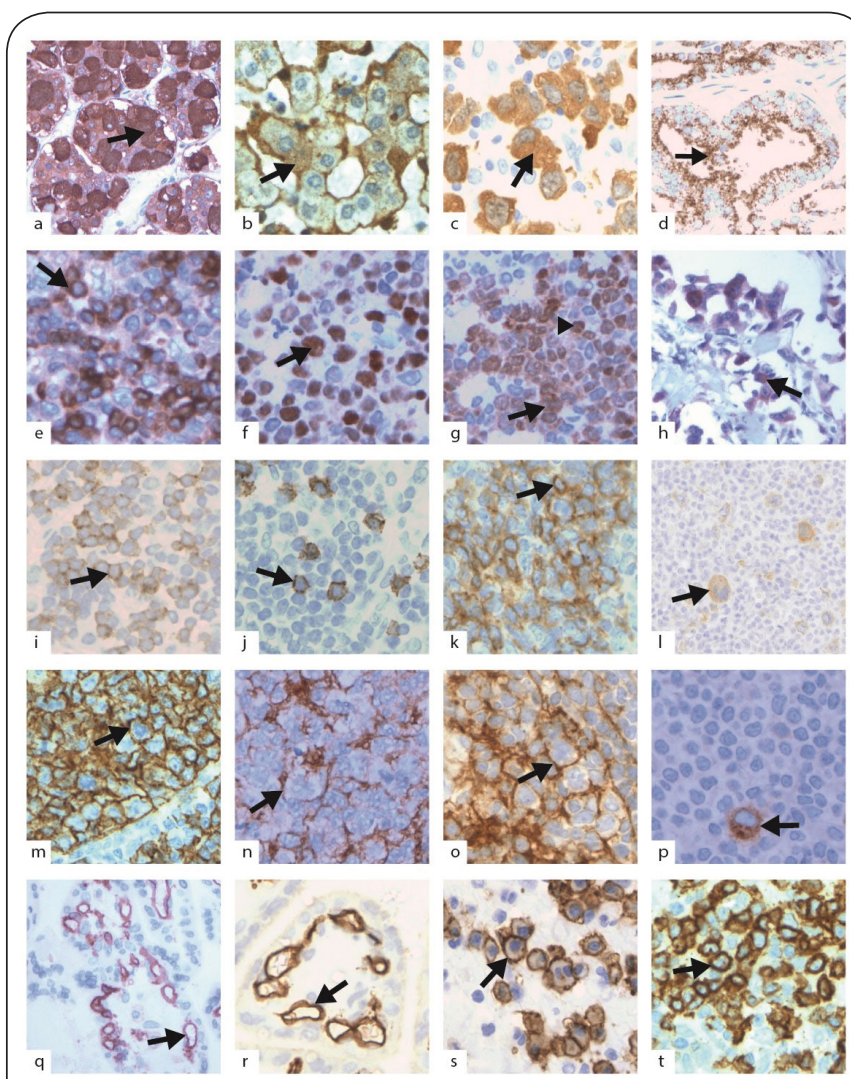

Figure 3. Immunostaining, magnification 400x for all panels. a) ACTH, cytoplasmic (arrow); b) Alpha-1- Fetoprotein, cytoplasmic (arrow); c) Alk, cytoplasmic (arrow); d) AMACR, cytoplasmic (arrow); e) Bcl 2, cytoplasmic (arrow); f) Bcl6, nucleus (arrow); g) BOB 1, cytoplasmic (arrow) and nuclear (arrow head $\mathbf{\Delta}$ ); h) Calcitonin, cytoplasmic (arrow); i) Cd7, membrane (arrow); j) Cd8, membrane (arrow); k) Cd10, membrane (arrow); l) Cd15, membrane (arrow); m) Cd20, membrane (arrow); n) Cd21, membrane (arrow); o) Cd 23 membrane (arrow); p) Cd30, membrane (arrow); q) Cd31, membrane (arrow); r) Cd34 membrane (arrow); s) Cd38, membrane (arrow); t) Cd57, membrane (arrow).

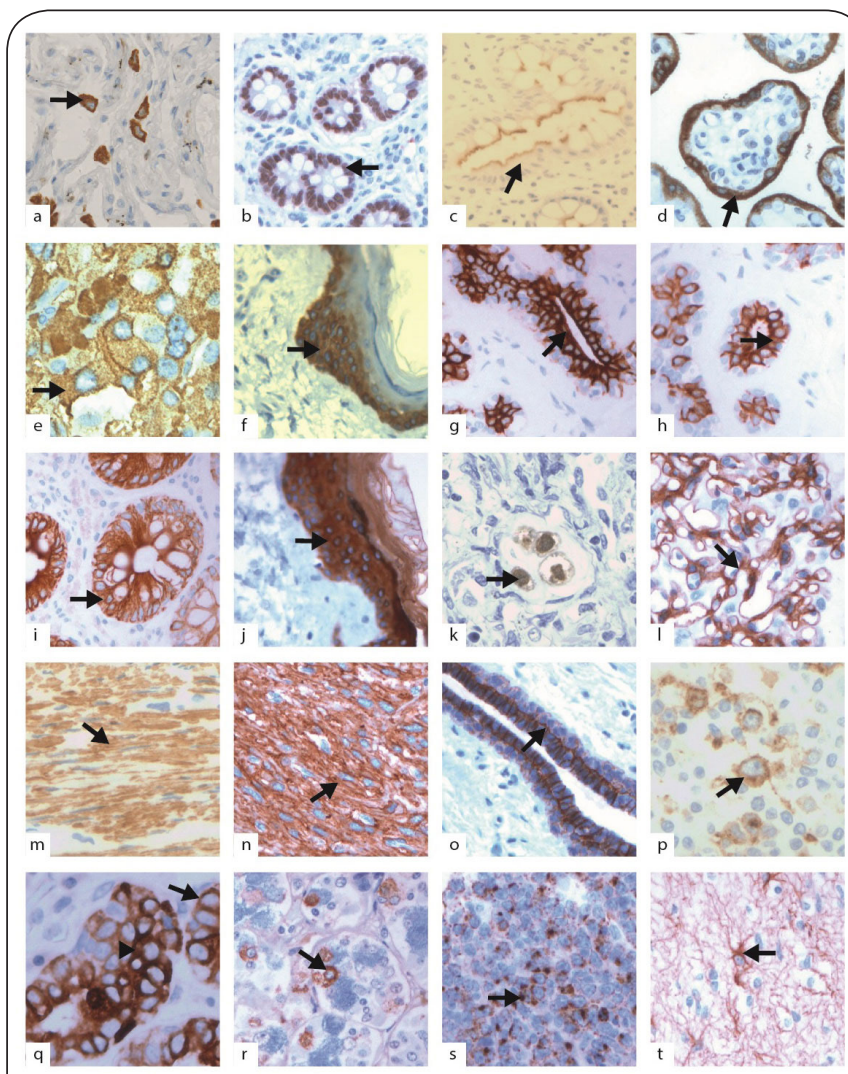

Figure 4. Immunostaining, magnification 400x for all panels. a) Cd68, cytoplasmic (arrow); b) Cdx2, nuclear (arrow); c) CEA, cytoplasmic (arrow); d) HCG, cytoplasmic (arrow); e) Chromogranin A, cytoplasmic (arrow); f) Ck 5/6, cytoplasmic (arrow); g) Ck 7, cytoplasmic (arrow); h) Ck8/18, cytoplasmic (arrow); i) Ck 20, cytoplasmic (arrow); j) CKPAN, cytoplasmic (arrow); k) CMV, nuclear (arrow); l) Collagen IV, membrane (arrow); m) Desmin, cytoplasmic (arrow); n) DOG-1, cytoplasmic (arrow); o) E-cadherin, membrane (arrow); p) EBV, cytoplasmic (arrow); q) EMA, membrane (arrow) and cytoplasmic ( $\boldsymbol{\Delta}$ arrow head); r) FSH, cytoplasmic (arrow); s) Granzyme B, cytoplasmic (arrow); t) GFAP, cytoplasmic (arrow). Abbreviation: Ck (Cytokeratin). tion from manual to automated IHC: first, it is imperative that at least one professional in the technical area be in charge of capturing, along with the experienced pathologist, the inadequacies in the marking obtained and making the appropriate change in the protocol. Second, knowing the demand of each antibody is critical in order to avoid previous dilution for an extended length of time, and on the platform tested, it is possible to keep $5 \mathrm{~mL}$ of diluted antibody. At the time of refilling of the diluted antibody, it is necessary to discard any dead volume present in the vial to avoid changing the antibody concentration determined by titration. Third, in centers where acquisition is complex or time-consuming, the management and purchase of reagents should be carefully planned given the importance of expiration dates of certain essential reagents (e.g., detection kits)—the equipment does not perform IHC until reagents with acceptable validity are recorded. Last, the use of purified water without contaminants is essential for the dilution of bulky fluids such as antigen retrieval buffers. While the use of purification stations for distilled water supply is an alternative, it requires periodic microbiological control.

The benefits of automation with IHC seem to meet the requirements of higher reproducibility, quality, and standardization concomitant with increased demand. Automation has been applied in many branches of the medical sciences, arriving definitively in pathology laboratories [16]. Among the innovations that are changing the practice in laboratories of pathological anatomy are genetic tests, telepathology, and adherence to automation [17]. Another form of innovation is the implementation of laboratory and bar code information 


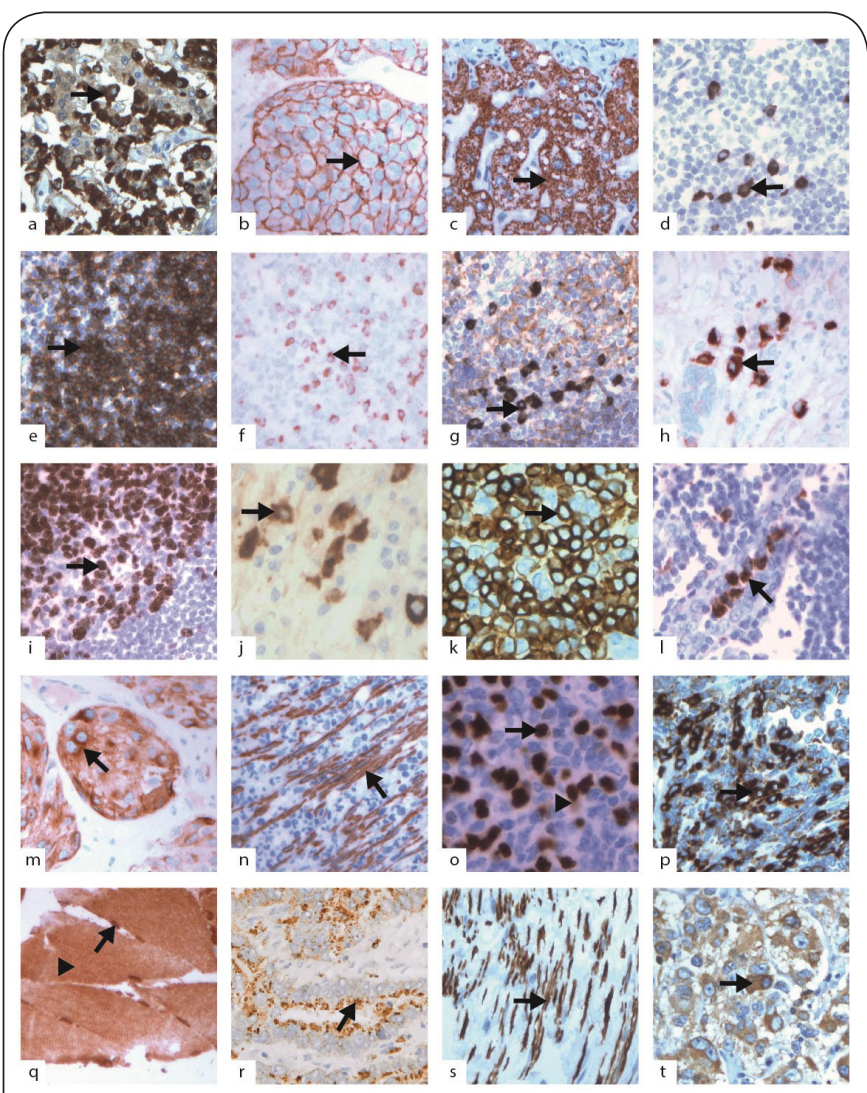

Figure 5. Immunostaining, magnification 400x for all panels. a) Glucagon, cytoplasmic (arrow); b) Her2, membrane (arrow); c) Hepatocyte, cytoplasmic (arrow); d) IgA, cytoplasmic (arrow); e) IgD, cytoplasmic (arrow); f) IgG, cytoplasmic (arrow); g) IgM, cytoplasmic (arrow); h) Kappa Light Chains, cytoplasmic (arrow); i) Ki-67, nuclear (arrow); j) LLC, cytoplasmic (arrow); k) LCA, membrane (arrow); l) Lysozyme, cytoplasmic (arrow); m) Melan-A, cytoplasmic (arrow); n) Muscle Actin, cytoplasmic (arrow); o) MUM 1, nuclear (arrow) and cytoplasmic ( $\boldsymbol{\Delta}$ arrow head); p) Myeloperoxidase cytoplasmic (arrow); q) Myoglobin, nuclear (arrow) and cytoplasmic ( $\boldsymbol{\Delta}$ arrow head); r) Napsin A, cytoplasmic (arrow); s) NFP, cytoplasmic (arrow); t) NSE, cytoplasmic (arrow).

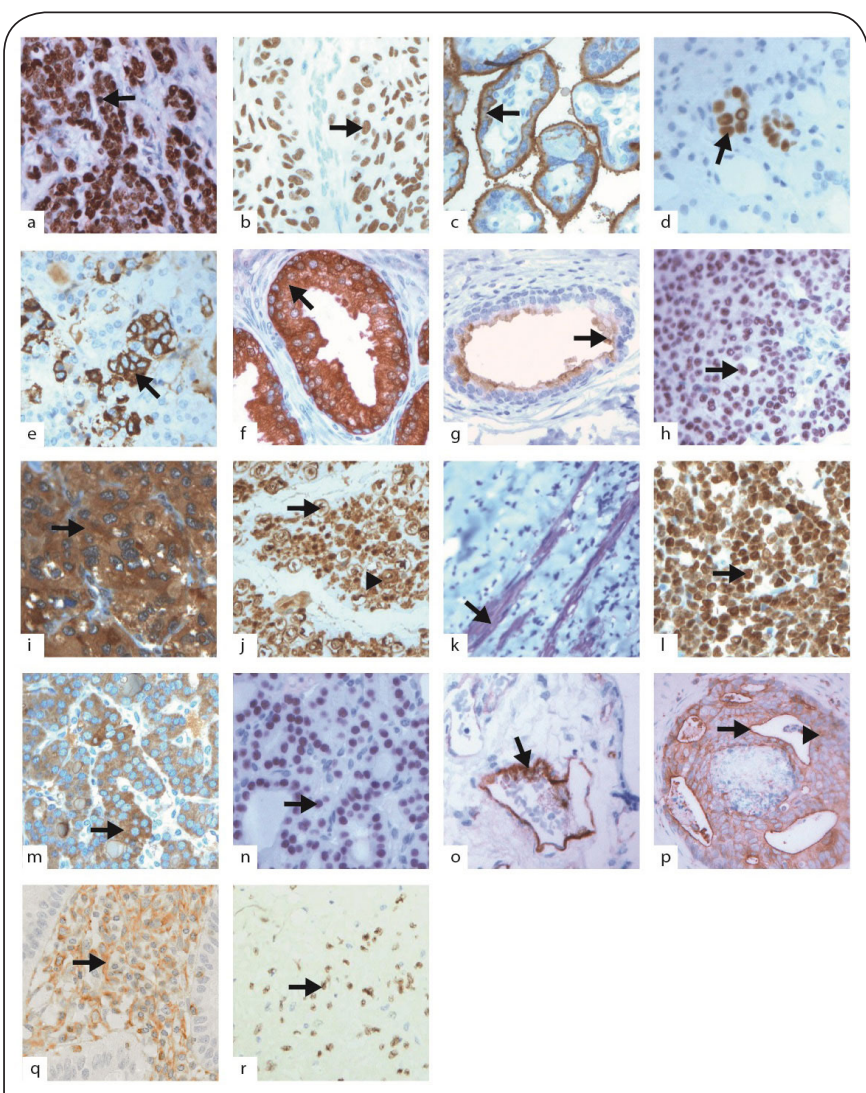

Figure 6. Immunostaining, magnification 400x for all panels. a) P53, nuclear (arrow); b) P63, nuclear (arrow); c) PLAP, cytoplasmic (arrow); d) Polioma Vírus, nuclear (arrow); e) Prolactin, cytoplasmic (arrow); f) PSA, cytoplasmic (arrow); g) PSMA, cytoplasmic (arrow); h) Estrogen Receptor, nuclear (arrow); i) Synaptophysin, cytoplasmic (arrow); j) S100, nuclear (arrow) and cytoplasmic ( $\boldsymbol{\Delta}$ arrow head); k) Smooth Muscle Actin, cytoplasmic (arrow); l) TDT, nuclear (arrow); m) Thyroglobulin, cytoplasmic (arrow); n) TTF1, nuclear (arrow); o) Von Willebrand Factor, cytoplasmic (arrow);

p) Villin, membrane (arrow) and cytoplasmic ( $\boldsymbol{\Delta}$ arrow head); q) Vimentin, cytoplasmic (arrow); r) WT1, nuclear (arrow). systems [18]. Promoting innovation in pathology is desirable; however, increasing costs with equipment and reagents are challenging and need to be overcome, the literature on the challenges in this area is scarce, and information on the subject of standardization, integration, and innovation in the laboratory often comes of other areas [17].

There are a few limitations of the study. First, there was an inability to compare immunostaining of concentrated antibodies with their ready-to-use counterparts. Because the main motivation of the study was the existence of antibody stocks in the laboratory, the laboratory did not acquire the equivalent ready-to-use stocks marketed by the manufacturer. The option to test automation in this laboratory was conditioned to the possibility of using the portfolio of pre-existing concentrated antibodies, which represented invested money. Second, no negative controls were used in the standardization, and the objective of the use of negative controls is to demonstrate that the reaction visualized is due to the interaction of the target protein epitope and the antibody parotope [19]. Last, a reaction cost analysis for manual and automated techniques was not performed.

Many factors make it impossible to establish a universal standard protocol for IHC, and it is up to each laboratory to choose and validate the protocol for each marker, whether the technique is automated or not. The standardization of an antibody that will be used in automation is part of the transition to automated IHC in open systems that allow the use of concentrated antibodies. In this case, the manufacturer's 


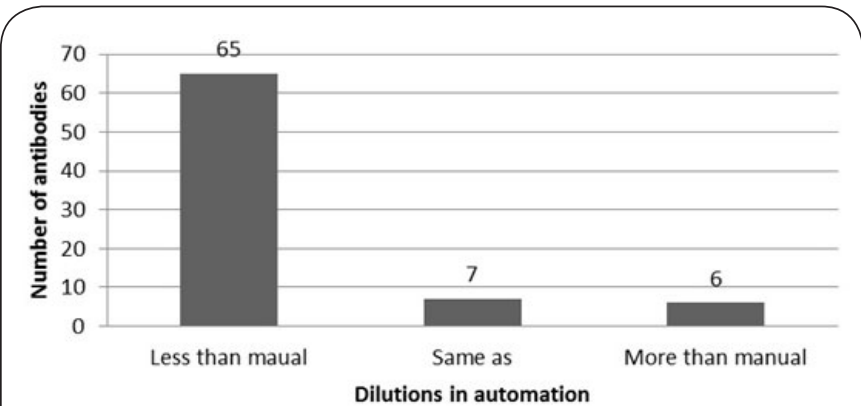

Figure 7. Antibody concentration used in automation in relation to manual technique.

recommendations are a good starting point $[\mathbf{7 , 2 0}]$.

\section{Conclusion}

If one is opting for an automation platform to perform immunohistochemistry according to the results presented here, it is possible to use concentrated antibodies that may already be in the laboratory, either for economic reasons or because a manufacturer does not have the antibody that is needed. For other equipment or antibodies, the standardization must be performed again. The process of standardization in large centers that have many antibodies can become an arduous task. However, with the possibility of migration to this incremental innovation, standardization should serve as a subsidy for a conscious migration in all areas of the laboratory, especially among pathologists, technicians, and administrators.

\section{Competing interests}

The authors declare that they have no competing interests.

\section{Authors' contributions}

\begin{tabular}{|l|c|c|}
\hline Authors' contributions & EFSP & CGZ \\
\hline Research concept and design & $\checkmark$ & $\checkmark$ \\
\hline Collection and/or assembly of data & $\checkmark$ & -- \\
\hline Data analysis and interpretation & $\checkmark$ & $\checkmark$ \\
\hline Writing the article & $\checkmark$ & -- \\
\hline Critical revision of the article & $\checkmark$ & $\checkmark$ \\
\hline Final approval of article & $\checkmark$ & $\checkmark$ \\
\hline Statistical analysis & $\checkmark$ & -- \\
\hline
\end{tabular}

\section{Publication history}

Editor: Khin Thway, The Royal Marsden Hospital, UK.

Received: 06-Feb-2020 Final Revised: 20-Apr-2020

Accepted: 16-May-2020 Published: 25-May-2020

\section{References}

1. Bogliolo L, Brasileiro Filho $\mathrm{G}$ and Barbosa AJA. Bogliolo patologia geral. 5ed. Rio de Janeiro: Guanabara Koogan. 2013.

2. McCourt CM, Boyle D, James J and Salto-Tellez M. Immunohistochemistry in the era of personalised medicine. J Clin Pathol. 2013; 66:58-61. | Article | PubMed

3. Moore DA, Young CA, Morris HT, Oien KA, Lee JL, Jones JL and Salto-Tellez $M$. Time for change: a new training programme for morpho-molecular pathologists? J Clin Pathol. 2018; 71:285-290. | Article | PubMed
Abstract | PubMed FullText

4. Okoye JO, Nnatuanya IN. Immunohistochemistry: A Revolutionary Technique in Laboratory Medicine. Clinical Medicine and Diagnostics. 2015; 5:60-69.

5. O'Hurley G, Sjostedt E, Rahman A, Li B, Kampf C, Ponten F, Gallagher $W M$ and Lindskog $C$. Garbage in, garbage out: a critical evaluation of strategies used for validation of immunohistochemical biomarkers. Mol Oncol. 2014; 8:783-98. | Article | PubMed Abstract | PubMed FullText

6. Yaziji $\mathrm{H}$ and Barry T. Diagnostic Immunohistochemistry: what can go wrong? Adv Anat Pathol. 2006; 13:238-46. | Article | PubMed

7. Taylor CR. Standardization in immunohistochemistry: the role of antigen retrieval in molecular morphology. Biotech Histochem. 2006; 81:3-12. | Article | PubMed

8. Kim SW, Roh J and Park CS. Immunohistochemistry for Pathologists: Protocols, Pitfalls, and Tips. J Pathol Transl Med. 2016; 50:411-418. I Article | PubMed Abstract | PubMed FullText

9. Taylor CR. The total test approach to standardization of immunohistochemistry. Arch Pathol Lab Med. 2000; 124:945-51. | Article I PubMed

10. Magaki S, Hojat SA, Wei B, So A and Yong WH. An Introduction to the Performance of Immunohistochemistry. Methods Mol Biol. 2019; 1897:289-298. | Article | PubMed Abstract | PubMed FullText

11. Prichard JW. Overview of automated immunohistochemistry. Arch Pathol Lab Med. 2014; 138:1578-82. | Article | PubMed

12. Gedda L, Bjorkelund $H$ and Andersson K. Real-time immunohistochemistry analysis of embedded tissue. Appl Radiat Isot. 2010; 68:2372-6. | Article | PubMed

13. Bogen, Steven A., and Seshi R. Sompuram. "A Linear Epitopes Model of Antigen Retrieval." In Antigen Retrieval Immunohistochemistry Based Research and Diagnostics, edited by Shan-Rong Shi and Clive R. Taylor, 168:287-302. Hoboken, NJ, USA: John Wiley \& Sons, Inc. 2010.

14. Vani K, Bogen SA and Sompuram SR. A high throughput combinatorial library technique for identifying formalin-sensitive epitopes. J Immunol Methods. 2006; 317:80-9. | Article | PubMed Abstract | PubMed FullText

15. Jaffe, Elaine Sarkin, Nancy Lee Harris, James Vardiman, Daniel A. Arber, and Elias Campo. Hematopathology E-Book. Elsevier Health Sciences. 2010.

16. Boenisch T. Anticuerpos, inmunohistoquímica básica, enzimología básica, métodos de coloración, controles, fondo. En: Métodos inmunohistoquímicos de coloración. 7ạ ed. USA: DAKO Corp. 2001; 5-15.

17. Hernandez JS, Dale JC, Bennet KE and Varkey P. Challenges and opportunities for medical directors in pathology and laboratory medicine: standardization, integration, and innovation. Am J Clin Pathol. 2010; 133:8-13. | Article | PubMed

18. Grimm EE and Schmidt RA. Reengineered workflow in the anatomic pathology laboratory: costs and benefits. ArCh Pathol Lab Med. 2009; 133:601-4. | Article | PubMed

19. Hewitt SM, Baskin DG, Frevert CW, StahI WL and Rosa-Molinar E. Controls for immunohistochemistry: the Histochemical Society's standards of practice for validation of immunohistochemical assays. J Histochem Cytochem. 2014; 62:693-7. | Article | PubMed Abstract | PubMed FullText

20. Cates JM and Troutman KA, Jr. Quality Management of the Immunohistochemistry Laboratory: A Practical Guide. App Immunohistochem Mol Morphol. 2015; 23:471-80. | Article | PubMed

Citation:

Pilar EFS and Zettler CG. Standardization of concentrated antibodies for use in automated immunohistochemistry. J Histol Histopathol. 2020; 7:4. http://dx.doi.org/10.7243/2055-091X-7-4 